\title{
Stellar proper motions in the Galactic bulge with ACS/WFC on HST
}

\author{
Will Clarkson ${ }^{1}$, Kailash Sahu${ }^{1}$, Jay Anderson ${ }^{2}$, T. Ed Smith ${ }^{1}$, \\ Thomas M. Brown ${ }^{1}$, Stefano Casertano ${ }^{1}$, R. Michael Rich ${ }^{3}$, Howard \\ E. Bond ${ }^{1}$, Timothy Brown ${ }^{4}$, Mario Livio ${ }^{1}$, Dante Minniti ${ }^{5}$, Nino \\ Panagia $^{1}$, Alvio Renzini ${ }^{6}$, Jeff Valenti ${ }^{1}$ and Manuela Zoccali ${ }^{5}$ \\ ${ }^{1}$ Space Telescope Science Institute, 3700 San Martin Drive, Baltimore, MD 21218, USA \\ email: clarkson@stsci.edu \\ ${ }^{2}$ Department of Physics and Astronomy, MS-108, Rice University, 6100 Main Street, Houston, \\ TX-77005, USA \\ ${ }^{3}$ UCLA, Los Angeles, California 90095-1562, USA \\ ${ }^{4}$ Las Cumbres Observatory Global Telescope, 6740 Cortona Dr. Suite 102, Santa Barbara, CA \\ 93117, USA \\ ${ }^{5}$ Universidad Catolica de Chile, Av, Vicuna Mackenna 4680, Santiago 22, Chile \\ ${ }^{6}$ INAF - Osservatorio di Padova, Vicolo dell'Osservatorio 5, 35122, Padova, Italy
}

\begin{abstract}
In 2004 the Sagittarius Window Eclipsing Extrasolar Planet Search (SWEEPS) project undertook a very deep ACS/WFC exposure-set of the Sgr-I low-reddening window in the Galactic Bulge, with repeat observations 2.04 years later. The combination of superb first-epoch sampling, wide field of view and high PSF stability of ACS/WFC on Hubble allows proper motions to be extracted for more than 137,000 objects, over 85,000 to accuracy better than

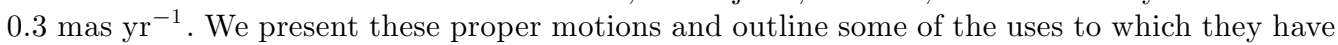
been put, including the separation of a pure-Bulge sample and the inner Galactic rotation curve.
\end{abstract}

Keywords. stars: kinematics, Galaxy: bulge, Galaxy: disk, Galaxy: kinematics and dynamics

\section{Introduction}

Proper motions have much to tell us about the dynamics and evolution of the Galactic Bulge. Constraints on the nature and spatial variation of the motion of stellar populations within the Bulge allow testing of dynamical galaxy models to a level not available for external galaxies. Equally importantly, stellar proper motions allow kinematic separation of a cleaner Bulge sample for studies of its age (Kuijken \& Rich 2002).

\section{Extraction of Relative Proper Motions from HST Photometry}

The first epoch observations included 265 exposures in F814W and 254 in F606W, at 339 seconds each and with subpixel dithers that well and redundantly sample pixel phasespace. In 2006, ten 345-second exposures in F814W were taken with a four-way sub-pixel dither. Mutual misalignment and rotation between epochs are of order 5 pixels and $<8^{\prime \prime}$ respectively. Up to 265 measurements are available for each star in 2004 using a modified version of the Anderson \& King routines; we combine the 2004-epoch measurements to produce a master catalogue from this epoch. Each position-estimate from 2006 is then mapped onto this 2004 master-list, producing up to ten proper motion estimates for each star. This mapping is computed separately for each star using 100-150 suitably selected nearby, well-measured objects. The proper motion error resulting is only $2{\text { mas } \mathrm{yr}^{-1} \text { at }}^{-1}$ 

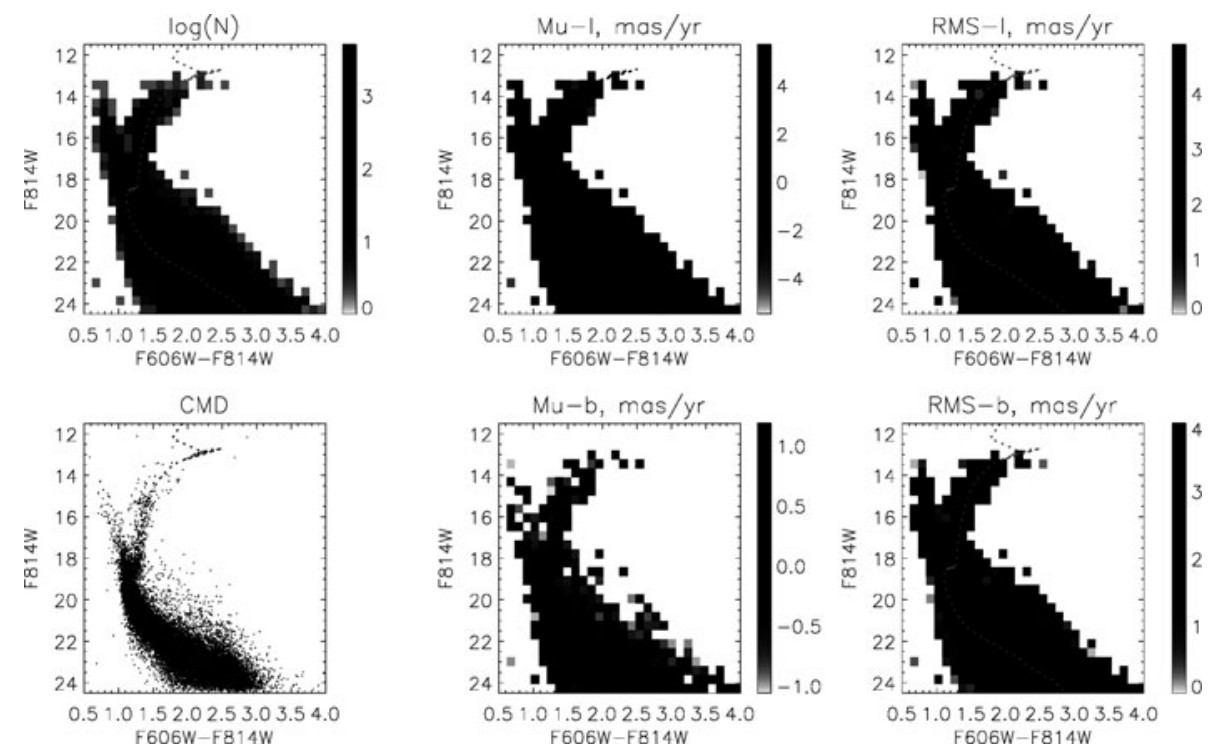

Figure 1. Proper motion Hess diagrams for our field, with mean-Bulge isochrone from SWEEPS photometry (Sahu et al. 2006). The Bulge is clearly visible in $\mu_{l}, \sigma_{b}$ and $\sigma_{l}$. Solar reflex motion contributes a relatively insignificant trend $\left|\mu_{l, b}\right|<0.2$ mas yr$^{-1}$ (e.g. Vieira et al. 2007)

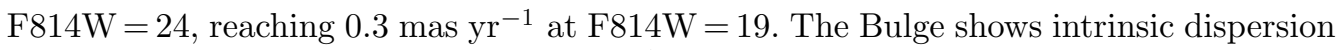

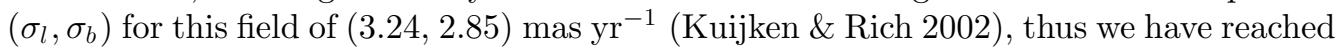
the precision necessary to observe real kinematic trends (Figure 1).

\section{Discussion}

Full uses of our unique dataset are described elsewhere (Clarkson et al. 2007 in preparation). By reference to the Bulge isochrone a photometric distance estimate can be assigned to main-sequence stars interior to the innermost spiral arm, averaging over the Bulge metallicity distribution with 500-1500 stars per distance-bin. This yields kinematic constraints as a function of distance, including the transverse rotation curve of the Milky Way down to $0.2 \mathrm{kpc}$ galactocentric radius. This indicates that the Bulge appears to only exhibit solid body-like rotation for galactocentric radii $\lesssim 0.7 \mathrm{kpc}$ (in qualitative agreement with Rich et al. 2007). A second product is the $\left\{v_{l}, v_{b}\right\}$ velocity ellipse as a function of distance, which will be used in future work to constrain dynamical models of the Bulge. Additionally, selecting stars by $\mu_{l}$ and $\sigma_{b}$ produces a clean sample of 21,292 probable Bulge objects, which will allow improved constraints on the Bulge age estimates.

\section{Acknowledgements}

This report is based on observations made with the Hubble Space Telescope, obtained at the Space Telescope Science Institute, operated by AURA, inc. under NASA contract.

\section{References}

Kuijken, K. \& Rich, R. M. 2002, AJ 124, 2054

Rich, R. M., Reitzel, D. B., Howard, C. D, \& Zhao, H-S. 2007, ApJ 658, L29

Sahu, K. C. et al., 2006, Nature 443, 534

Vieira, K. et al., 2007 AJ 134, 1432 